\title{
Variation, Signal, and Noise in Cerebellar Sensory-Motor Processing for Smooth-Pursuit Eye Movements
}

\author{
Javier F. Medina and Stephen G. Lisberger \\ Howard Hughes Medical Institute, Department of Physiology, W. M. Keck Foundation Center for Integrative Neuroscience, University of California, San \\ Francisco, California 94143-0444
}

\begin{abstract}
Neural responses are variable, yet motor performance can be quite precise. To ask how neural signal and noise are processed in the brain during sensory-motor behavior, we have evaluated the trial-by-trial variation of Purkinje cell (PC) activity in the floccular complex of the cerebellum, an intermediate stage in the neural circuit for smooth-pursuit eye movements. We find strong correlations between small trial-by-trial variations in the simple spike activity of individual PCs and the eye movements at the initiation of pursuit. The correlation is lower but still present during steady-state pursuit. Recordings from a few pairs of PCs verified the predictions of a model of the PC population, that there is a transition from highly covariant $\mathrm{PC}$ activity during movement initiation to more independent activity later on. Application to the data of a theoretical and computational analysis suggests that variation in pursuit initiation arises mostly from variation in visual motion signals that provide common inputs to the PC population. Variation in eye movement during steady-state pursuit can be attributed primarily to signal-dependent motor noise that arises downstream from PCs.
\end{abstract}

Key words: flocculus; correlation; noise reduction; cerebellum; motor control; visual motion

\section{Introduction}

Even Tiger Woods cannot replicate the same perfect golf swing over and over. Some motor variation is inevitable (Todorov and Jordan, 2002; Davids et al., 2006). Still, given the amount of noise in the operation of individual neurons (Calvin and Stevens, 1967; White et al., 2000), and the conspicuously variable responses of individual neurons to identical presentations of the same stimulus (Calvin and Stevens, 1968; Softky and Koch, 1993; Shadlen and Newsome, 1994; Stein et al., 2005), movements can be remarkably precise. To achieve precise movements, the brain must reduce neural noise at each processing level (e.g., by pooling or averaging the activity of many upstream neurons) (Shadlen and Newsome, 1994; Pouget et al., 2000; van Beers et al., 2002; Carmena et al., 2005; Averbeck et al., 2006). At the same time, noise will be added at each level because of the probabilistic nature of synaptic transmission and action potential generation (Calvin and Stevens, 1967; White et al., 2000). How the brain achieves "reliable computation with unreliable components" (von Neumann, 1956) will depend on how much noise is added and reduced at each processing level, and ultimately on the trial-to-trial variation of neural responses.

We can summarize the factors that contribute to the trial-to-

Received March 24, 2007; revised April 27, 2007; accepted May 14, 2007.

This work was supported by the Howard Hughes Medical Institute and National Institutes of Health Grants NS34835 and EY017210. We thank many people for helpful comments on previous versions of this manuscript. We are grateful to Scott Ruffner for computer programming; to Karen MacLeod, Elizabeth Montgomery, and Stefanie Tokiyama for their attention to our monkeys; to Laszlo Bocskai and Ken McGary for technical assistance; and to Dirk Kleinhesselink and David Wolfgang-Kimball for computer system administration.

Correspondence should be addressed to Dr. Javier F. Medina, Department of Physiology, Box 0444, 513 Parnassus Avenue, Room HSE-808, University of California, San Francisco, CA 94143-0444. E-mail: jmedina@phy.ucsf.edu. D0I:10.1523/JNEUROSCI.1323-07.2007

Copyright $\odot 2007$ Society for Neuroscience $\quad$ 0270-6474/07/276832-11\$15.00/0 trial variation in the spike rate of any individual neuron in terms of two components: correlated variation that is shared across a population of neurons versus independent noise that is private to each individual neuron. Averaging the activity of many neurons will reduce independent noise to a degree that depends on the size of the neuronal population, but correlated variation cannot be "averaged away" (Shadlen and Newsome, 1994; Abbott and Dayan, 1999; Averbeck et al., 2006). If a population of sensory neurons with some correlated variation provided a strong common input to multiple neurons at the next level, then the activity of the downstream neurons would also covary from trial to trial. The resulting correlated component of neural responses would propagate inexorably onward, ultimately leading to motor variation in the behavior.

In the present study, we have developed and used a new approach to examine how neural signals and noise are processed by the brain during sensory-motor behavior. Based on an analysis of the trial-by-trial correlations between neural and behavioral responses, we examine the neural sources of motor variation upstream and downstream of the floccular complex of the cerebellum, an intermediate stage in the neural circuit that drives smooth-pursuit eye movements (Robinson and Fuchs 2001; Thier and Ilg, 2005). Our data reveal surprisingly strong trial-bytrial correlations between the variation in pursuit eye movements and the responses of individual Purkinje cells (PCs) during the initiation of pursuit, as well as remarkably small amounts of variance reduction between the responses of individual PCs and eye movement behavior. Estimates of the variance of noise added downstream from the cerebellum and the trial-by-trial correlations between the firing of pairs of PCs support the hypothesis that most of the variation in the initiation of pursuit arises upstream from the cerebellum, presumably from noise in visual 


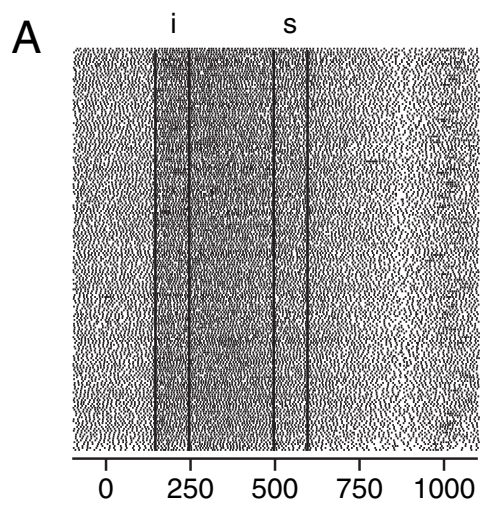

Time from target motion onset $(\mathrm{ms})$

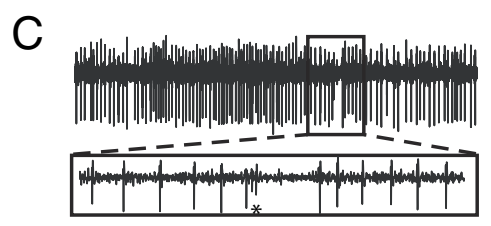

nals proportional to horizontal and vertical eye position (Fuchs and Robinson, 1966), which then were passed through an analog circuit to create signals proportional to horizontal and vertical eye velocity. The circuit differentiated frequency content from 0 to $25 \mathrm{~Hz}$ and filtered higher frequencies with a roll-off of $20 \mathrm{~dB}$ per decade. Analog signals were digitized at $1 \mathrm{kHz}$; the digital records of eye velocity were differentiated to obtain the eye acceleration.

Single-unit recording. Recordings from single PCs were made with glass-insulated platinumiridium microelectrodes manufactured in our laboratory. Recordings from pairs of PCs were made with glass-insulated tungsten microelectrodes purchased from Alpha Omega Technologies (Brielle, NJ) and were driven into the brain using a four-tower NAN drive from Plexon (Dallas, TX). The approach to the floccular complex was the same as has been described previously (Stone and Lisberger, 1990). PCs could be distinguished from all the other neuron types in the cerebellar cortex by the presence of occasional complex spikes (see Fig. 1C). The recorded extracellular unit activity was passed through a standard head stage, amplified, filtered (bandpass, $100 \mathrm{~Hz}$ to $10 \mathrm{kHz}$ ), and converted into trigger pulses off-line under experimenter control using a spike sorter/discriminator. We verified that the recordings were from single PCs by viewing records digitized at $25 \mathrm{kHz}$ and checking that there were no instances in which two spikes occurred within a refractory period.

motion processing (Osborne et al., 2005). Motor noise is added downstream of the cerebellum (Jones et al., 2002; van Beers et al., 2004) but makes a significant contribution to motor variation only later in the response.

\section{Materials and Methods}

Eye movements were recorded from two rhesus monkeys (Macaca mulatta) that had been trained and prepared for experiments using methods described previously (Medina et al., 2005; Ramachandran and Lisberger, 2005). During each experimental session, the monkey sat in a dimly lit room inside a specialized primate chair with its head immobilized and received a water reward for accurately tracking a small spot presented on a screen in front of it. While the monkey was tracking, we recorded the activity of well isolated PCs in the right floccular complex of the cerebellum. The Institutional Animal Care and Use Committee at the University of California, San Francisco had approved all procedures.

Pursuit task. The monkey normally completed $\sim 1200$ pursuit trials in a session. At the start of each trial, a stationary target appeared at the center of the screen, and the monkey was required to fixate it for an interval that was randomized between 600 and $1200 \mathrm{~ms}$. To minimize the occurrence of saccades during the initiation of pursuit, target trajectories followed standard step/ramp motion (Rashbass, 1961); after the fixation period, the target was displaced eccentric to the position of gaze (step) and immediately began moving toward the fixation point at constant speed (ramp) for a total duration of $750 \mathrm{~ms}$. If the monkey kept gaze within a $2 \times 2^{\circ}$ window around the moving target, then he received a water reward at the end of the trial. The direction of target motion for each particular trial was either in the $\mathrm{ON}$ direction of the PC being recorded (normally RIGHT or DOWN) or in the OFF direction (normally LEFT or UP), although only the data for the ON direction were subjected to the analyses described later. The speed of the target was 10 , 20 , or $30 \%$ s. Both direction and speed were varied randomly from one trial to the next so that the monkey could not anticipate the target trajectory.

The magnetic search coil technique was used to obtain real-time sig-
Data analysis. Eye velocity and extracellular unit activity were analyzed after each experiment using an interactive computer program. The horizontal and vertical eye velocity traces from individual trials were displayed on the computer screen, and all saccades were excised manually. Any behavioral or neural data in the interval between the start and the end of the rapid eye deflections associated with each saccade was treated as missing. To evaluate the millisecond-by-millisecond correlation between the firing rate and the behavior, we started by reducing the three dimensions of eye movement to a single dimension and converting it to the same units as firing rate. For each PC, we computed the inverse model that relates average firing rate to the average eye position, velocity, and acceleration (see Eq. 1 in Results). We then used the average inverse model to predict the firing rate as a function of time for each trial that contributed to the average. To obtain a comparable index of the firing rate of the PC, we used a reciprocal of the interspike interval algorithm to convert the spike train for each individual pursuit trial to a continuous firing rate variable (Lisberger and Pavelko, 1986).

We calculated the correlation matrix after averaging the predicted and actual firing rates in $20 \mathrm{~ms}$ bins. Thus, the value of the correlation matrix at $\left(t_{1}, t_{2}\right)$ indicates the correlation coefficient between two separate arrays, each one comprising as many elements as there were trials for a given speed of target motion in the ON direction. Each element in the first array contained the average value of the inverse model prediction in the interval $\left[t_{1}-9, t_{1}+10\right]$ for a particular trial, and the corresponding element in the second array contained the average value of the actual firing rate in the interval $\left[t_{2}-9, t_{2}+10\right]$ for the same trial. To assess the significance of the correlation, we bootstrapped to compute "control" correlation matrices in which the elements of the arrays (i.e., the trials) were shuffled randomly. We also verified that the correlation matrix did not change materially when we used non-overlapping sets of trials to compute the inverse model and the neuron-behavior correlation.

\section{Results}

We recorded neural and behavioral responses simultaneously as monkeys moved their eyes to track repeated presentations of a 
target that was initially stationary and then moved at constant speed on a display in front of them. The raster in Figure $1 \mathrm{~A}$ shows the responses of an example PC to 200 repetitions of the same stimulus. The raw record in Figure $1 C$ shows an example of a complex spike response from this recording (asterisk), verifying that it came from a PC. Figure $1 A$ reveals a vigorous and consistent pattern of spiking from trial to trial. Yet, analysis of the variance reveals considerable trial-by-trial variation in the firing rate of the PC and in the eye position, velocity, and acceleration in the same trials (Fig. $1 B$ ). The SD of each parameter of eye motion (thin traces) was small during fixation, increased quickly during the first $100 \mathrm{~ms}$ of the response as the eyes accelerated to match the speed of the target, and remained high and fairly constant throughout the first $500 \mathrm{~ms}$ of pursuit, the interval we used for most of our quantitative analyses. The time course of behavioral variation was similar for all 51 recording sessions. Typical SDs of the smooth component of eye motion during the initiation of pursuit (interval marked “i”) were as follows: eye position, $0.3-0.6^{\circ}$; eye velocity, $2-3 \%$; eye acceleration, $40-70 \% \mathrm{~s}^{2}$.

\section{Neuron-behavior correlations during pursuit}

Before computing trial-by-trial correlations between neural and behavioral responses, it was necessary to convert our measurements of smooth eye movement into the same units as firing rate and to reduce the three dimensions of eye movement (position, velocity, and acceleration) to the single dimension of firing rate. For each $\mathrm{PC}$, we computed the average firing rate across trials on a millisecond-by-millisecond basis and used a regression model developed by others to fit the average firing rate as a weighted, linear combination of the average eye position, velocity, and acceleration (Shidara et al., 1993; Leung et al., 2000; Roitman et al., 2005):

$$
f r(t)=a \ddot{E}(t-\Delta t)+r \dot{E}(t-\Delta t)+k E(t-\Delta t)+r r
$$

where $r r$ is the spontaneous firing rate during fixation and $\Delta t$ indicates by how much time the eye movement averages need to be shifted to optimize the fit to the average firing rate $(-8 \mathrm{~ms}$ for the PC analyzed in Figs. 1 and 2). The values of parameters $a, r$, and $k$ represent the average sensitivity of the PC to eye acceleration $\left(0.19\right.$ spikes/s per degrees $\left./ \mathrm{s}^{2}\right)$, velocity $(0.68 \mathrm{spikes} / \mathrm{s}$ per degrees/s), and position $(-0.39$ spikes/s per degree $)$. The terms $a \ddot{E}(t), r \dot{E}(t)$, and $k E(t)$ represent the contributions of average acceleration, velocity, and position to the average firing rate (Fig. $2 \mathrm{~A}$, bottom plot). As shown in detail below, the regression model provided an excellent fit to the average firing rate during both the initiation and the steady-state phases of the pursuit response, for this and all of the other 51 PCs in our sample. As reported previously (Takemura et al., 2001), more elaborate models with acceleration-dependent nonlinearities, or extra sensory terms related to visual signals, produced only marginally better fits. Their use did not alter any of our conclusions.
B
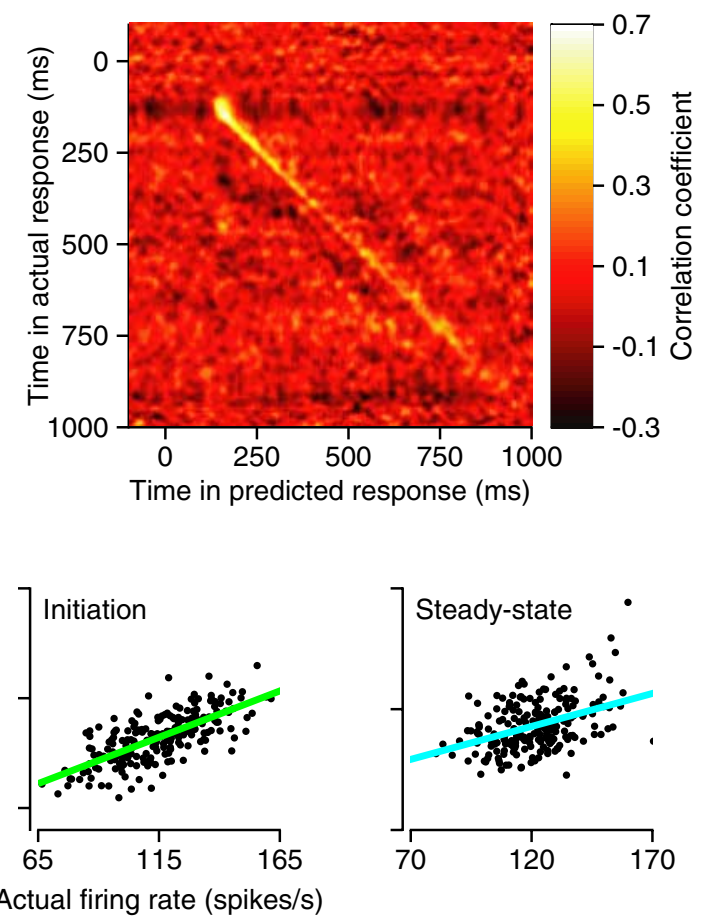

Actual firing rate (spikes/s)

Figure 2. Correlation between eye movements and the firing rate of an example PC. $\boldsymbol{A}$, The top two sets of traces show actual shows the contributions of the average eye position (Pos), velocity (Vel), and acceleration (Accel) to the average predicted firing data from a different trial. Colored lines show the result of linear regression analysis. The labels $\mathrm{f}, \mathrm{i}$, and $\mathrm{s}$ indicate specific times within the intervals indicated by the same indices in Figure 1.

Equation 1 is normally used to predict the time-varying average firing rate from the time-varying averages of eye acceleration, velocity, and position, but in our analysis, we used it to obtain a surrogate of the eye movement for each trial in the units of firing rate (spikes/s). We used Equation 1 and the values of $a, r, k$, and $r r$ from the average model to convert the time-varying eye acceleration, velocity, and position traces in each trial into a prediction of the firing rate in that trial (Fig. $2 \mathrm{~A}$, middle plot, the "predicted firing rate").

During the initial pursuit response, we found a strong correlation between the trial-by-trial variations in predicted and actual firing rate of PCs. The color of each pixel in the correlation matrix of Figure $2 B$ indicates the trial-by-trial correlation coefficient $(R)$ at the corresponding pair of time points along the $x$ - and $y$-axes. $R$ reached values as high as 0.7 for this particular PC, as shown by the white pixels in the center of the yellow blob in the top left quadrant of the matrix. A more conventional view of the correlation is shown in Figure $2 C$ by plotting predicted versus actual firing rate for all individual trials in three separate scatter plots that represent data from three time points (Fig. $2 \mathrm{~A}, \mathrm{f}, \mathrm{i}$, and $\mathrm{s}$ ). The correlation is almost nonexistent during fixation ( $f, r=$ $0.08)$, strongest during the initiation of pursuit (i, $r=0.68)$, and weaker during steady-state pursuit ( $s, r=0.29$ ). Because the prediction of the model defined by Equation 1 is linearly related to the eye movements that comprise the behavior, we use the term "neuron-behavior correlation" or " $R_{N B}$ " to describe the correlations outlined in Figure 2, $B$ and $C$. For the same reason, we use the predicted firing rate as an index of the eye movement 

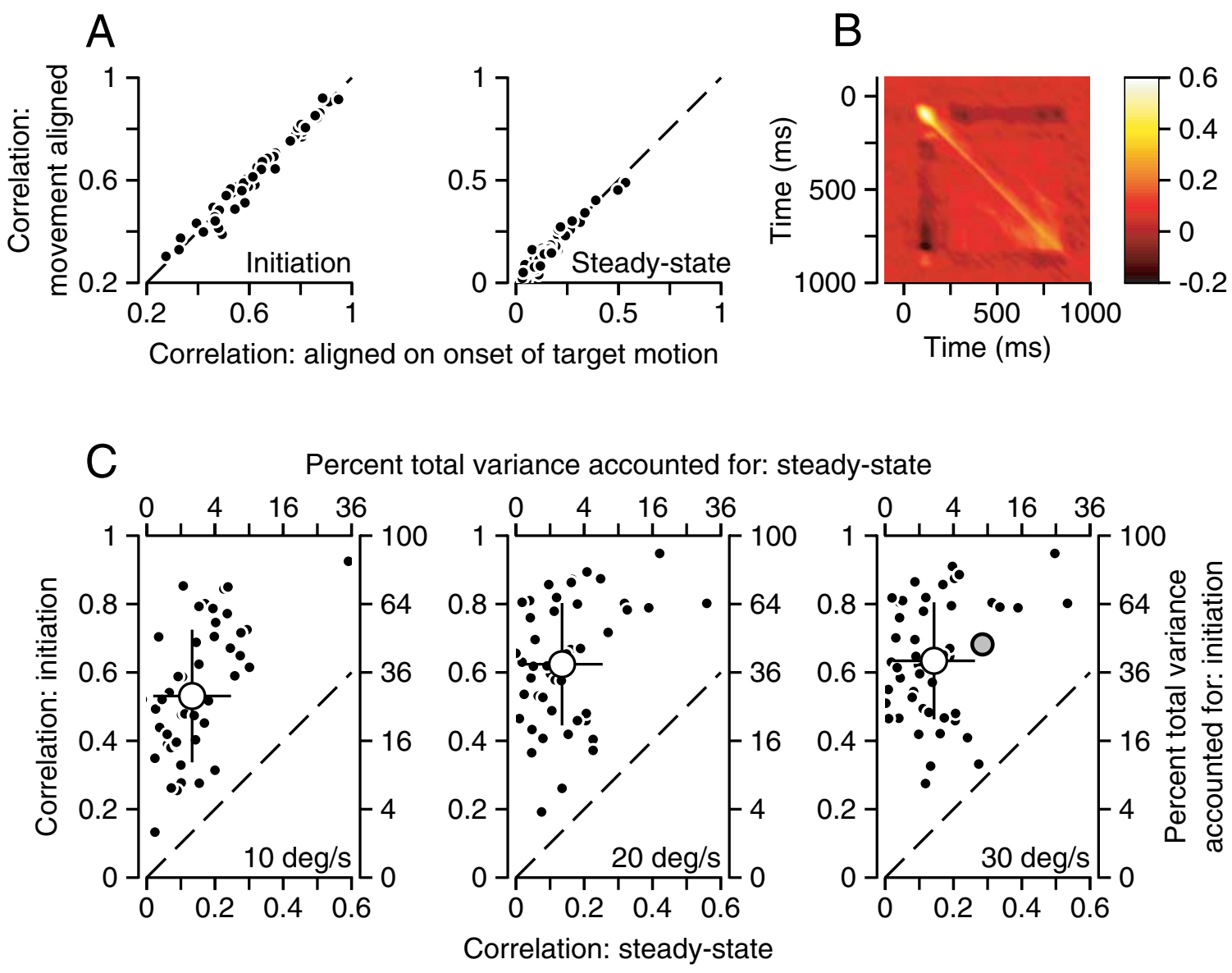

Figure 3. Summary of neuron-behavior correlations for the population of floccular PCS. $A$, Comparison of $R_{N B}$ when the trials were aligned on the onset of target motion versus the onset of eye motion. Each point shows data for a single PC, and the two graphs show measures made during the initiation or steady state of pursuit. $B$, Correlation matrix like that described in Figure $2 B$, averaged across the sample of $51 \mathrm{PCS}$. C, The three graphs compare $R_{N B}$ during the initiation versus the steady state of pursuit for target motion at three speeds $\left(10,20\right.$, and $30^{\circ} / \mathrm{s}$ from left to right). Each small filled circle shows the response for one PC. The large open symbol with error bars shows the mean and SD across the sample. The gray filled symbol in the right graph shows the example PC from Figures 1 and 2. The dashed lines have slopes of 1 . The bottom and left axes indicate the value of the correlation coefficient $\left(R_{N B}\right)$, and the top and right axes indicate the percentage of eye movement variance accounted for by firing rate $\left(R_{N B}{ }^{2}\right)$.

and its variation and refer to it in our equations with the subscript "EYE", even though it has the same units as firing rate.

All 51 PCs in our sample had neuron-behavior correlations with features very similar to those of the example PC shown in Figure 2. In the correlation matrices for the example PC (Fig. $2 B$ ) and the population average across all 51 PCs (Fig. 3B), the region of statistically significant neuron-behavior correlation appears as a thin band running more or less along the diagonal from top left to bottom right. Thus, the temporal window of the correlations was brief: they were local in time. There also is a period of negative correlations extending both to the right and down from the time of the highest correlation, during the initiation of pursuit. We attribute these features to negative correlations in the autocorrelation matrices for eye velocity and eye acceleration (supplemental Fig. 1, available at www.jneurosci.org as supplemental material). Correlations were retained in the face of crossvalidation analysis and were lost when the eye movements and firing rates were shuffled across trials (see Materials and Methods).

Figure $3 C$ shows on a neuron-by-neuron basis that the neuron-behavior correlation was consistently larger during the initiation of pursuit than later in the response: each PC plots well above the unity line. The average value of $R_{N B}$ during the initiation of pursuit across the population of 51 PCs was slightly $<0.6$ when target speed was $10 \%$ and $>0.6$ when target speed was 20 or $30 \%$. The average value of $R_{N B}$ during steady-state pursuit was $\sim 0.12$ for all speeds of target motion.

Several trivial explanations do not seem to be able to account for the differences in $R_{N B}$ between the initial and the steady-state components of the pursuit response. First, the value of $R_{N B}$ did not depend on whether the correlation matrix was computed with all traces aligned on the onset of target motion, or after shifting each neural/behavioral response in time so that all eye movement traces were aligned on the moment of the initiation of pursuit (Fig. 3A). This rules out the uninteresting possibility that $R_{N B}$ is higher during pursuit initiation simply because of slight trial-by-trial variation in the timing of the onset of pursuit. Second, the difference in the correlation coefficient between initiation and steady-state pursuit cannot be attributed to systematic differences in the variance of either the neural or the behavioral responses across phases of pursuit, because these differences were small and not consistently higher or lower for any particular phase of the pursuit response across our sample of PCs (Fig. 1C). Third, the lower values of $R_{N B}$ during steady-state pursuit cannot be attributed to a worse fit of the regression model of Equation 1 during steady-state pursuit, because the fits were equally good in both the initiation and steady-state phases (Fig. 4A,D). Linear regression analysis of the average firing rate of the model versus 


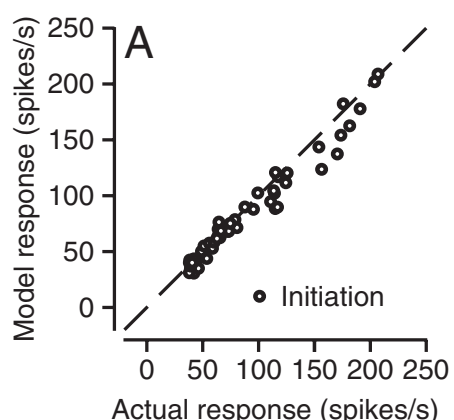

Actual response (spikes/s)

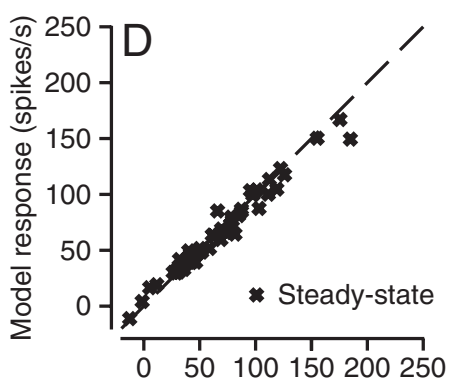

Actual response (spikes/s)
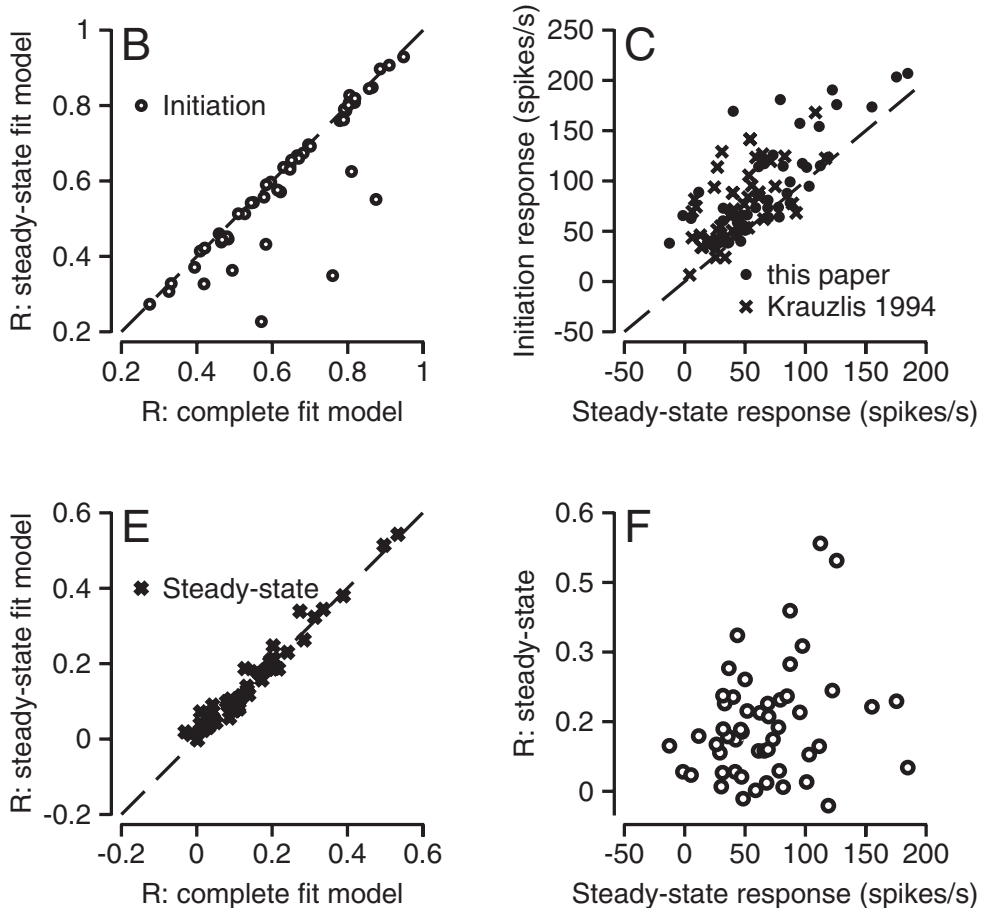

Figure 4. Control analyses to show that the low values of neuron- behavior correlation during steady-state pursuit are not artifacts. In all graphs, each point indicates data for one PC. $\boldsymbol{A}, \boldsymbol{D}$, The average response of the inverse model is plotted as a function of that measured from the actual data for the initiation $(\boldsymbol{A})$ and steady-state $(\boldsymbol{D})$ phase of the pursuit response. $\boldsymbol{B}, \boldsymbol{E}$, The neuron-behavior correlation measured using a model fitted only to the steady-state component of the response is plotted as a function of that measured using a model fitted to the entire response, for the initiation $(\boldsymbol{B})$ and steady-state $(\boldsymbol{E})$ phase of the response. The model was fitted to the steady state by excluding data from the first $300 \mathrm{~ms}$ after the onset of target motion. $\boldsymbol{C}$, The amplitude of the neural response during the initiation of pursuit is plotted as a function of that during steady-state pursuit; filled circles and $x$ symbols show data from this study and from Krauzlis and Lisberger (1994). $\boldsymbol{F}$, The neuron- behavior correlation during steady-state pursuit is plotted as a function of the magnitude of the steady-state response. All neural responses were plotted after subtracting the background firing rate during fixation. Data are for target motion at $30 \mathrm{deg} / \mathrm{s}$.

the actual average firing rate across all PCs revealed regression slopes of 0.936 and 0.951 in the initiation and steady-state intervals, with correlation coefficients of 0.98 in both cases (Fig. $4 A, D)$. Furthermore, the value of $\mathrm{R}_{N B}$ during steady-state pursuit did not increase when the regression model was fitted only to the steady-state phase of the behavior (Fig. $4 E$ ), whereas that during the initiation of pursuit decreased in seven PCs (Fig. $4 B$ ). Finally, we cannot explain the low values of $R_{N B}$ during steady-state pursuit as a consequence of selection bias or a failure to sample PCs with a strong response during this phase of the movement. We recorded from all PCs that were responsive, during the initiation, the steady-state, or both phases of the pursuit response. Our sample shows the same range of response amplitudes during the initiation and steady-state of pursuit as found in a previous study from our laboratory (Krauzlis and Lisberger, 1994) (Fig. 4C), and the relationship between the value of $R_{N B}$ and response amplitude during steady-state pursuit (Fig. $4 F$ ) gives no evidence that neurons with small responses that we may have failed to sample would yield large values of $R_{N B}$.

To quantify the contribution of $R_{N B}$ to the total variance in firing rate as a function of time, we selected the $45^{\circ}$ diagonal (top left to bottom right) through the correlation matrix that had the largest summed value of correlations and measured the value of $R_{N B}$ at each time along that diagonal: Figure $5 A$ shows $R_{N B}$ as a function of time along the diagonal selected for the example PC in Figure $2 B . R_{N B}{ }^{2}(t)$ estimates the fraction of the variation in PC firing that could be called "signal" in the sense that it could be attributed to variation in the eye movement at each time. Figure $5 B$ shows the time course of the total variance in the firing rate of the example PC (bold trace) and the fraction that was related to the evoked eye movement (gray fill). During the initiation of pursuit, $\sim 50 \%$ of the variation in the firing rate of this PC was related to the evoked eye movement. During steady-state pursuit, $<10 \%$ of the variation in the firing rate of this PC could be attributed to variation in the evoked eye movement. Because the predicted firing rate is linearly related to the smooth eye movement vector, the converse statement also is true: during the initiation and steady-state phases of pursuit, $\sim 50 \%$ and $<10 \%$ of the variation in the evoked eye movement could be attributed to variation in the firing of this one PC.

Figure $5 C$ provides a population summary of the time course of the signal-to-noise ratio of PC firing, defined as the percentage of total firing rate variance attributed to the trial-by-trial variation in the smooth eye movement divided by that not related to eye movement. The average time course of the signal-to-noise ratio for all $51 \mathrm{PCs}$ showed a pronounced peak of $\sim 1.0$ during the initiation of pursuit and rapidly dwindled to 0.1 (Fig. $5 C$, bold trace). A similar time course was seen in each individual PC (Fig. $5 C$, gray traces)

\section{Time courses of neuron-neuron correlations and variance of} downstream noise

Our measurements of neuron-behavior correlations have important implications regarding how signals and noise are processed as they move through the sensory-motor circuit for pursuit. As pointed out in the Introduction, two important factors control how well the brain generates precise movements in the face of the noisiness of individual neurons: the degree of neuronneuron correlation, which determines how much noise reduction can be achieved by averaging the activity of neuronal popu- 
lations; and the amount of neural noise that is added at each processing level. The strong neuron-behavior correlations found during the initiation of pursuit suggest two intuitions about these variables. First, if the variation in the firing rate of each of any two PCs of a large population is highly correlated with the variation in the emitted behavior on a trial-by-trial basis, then the firing rates of the two PCs also should be correlated with each other (this intuition breaks down if the number of PCs contributing to the behavior is very small). Second, because more downstream noise will lead to lower values of neuron-behavior correlation, a high neuron-behavior correlation implies that the amount of noise added downstream is relatively small. The mathematical analysis and computer simulation described in the Appendix formalize these two intuitions. If we assume that the responses of more than $\sim 40$ PCs are averaged to generate a motor command, then the average neuron-neuron correlation and the average variance of downstream noise can be approximated by the following equations:

$$
\begin{aligned}
& R_{N N}(t)=R_{N B}(t) \sqrt{\frac{\sigma_{E Y E}^{2}(t)}{\sigma_{F R}^{2}(t)}} \\
& \frac{\sigma_{B S}^{2}}{\sigma_{F R}^{2}}(t)=\frac{\sigma_{E Y E}^{2}}{\sigma_{F R}^{2}}(t)-R_{N N}(t)
\end{aligned}
$$

where $R_{N N}$ and $R_{N B}$ are the average values of neuron-neuron and neuron-behavior correlations across our sample and the $\sigma_{X}^{2}$ are the average variances of the firing rate $\left(\sigma_{F R}^{2}\right)$, the surrogate of eye movement created by the predictions from the inverse model of Equation $1\left(\sigma_{E Y E}^{2}\right)$, and the noise added downstream $\left(\sigma_{B S}^{2}\right)$. The variances are all expressed as a ratio, normalized to the average variance of $\mathrm{PC}$ firing rate, to emphasize that the variance ratio on the right side of the equations is an index of noise reduction between the PCs and the evoked movement. We note that the ability to estimate the noise reduction depends on using Equation 1 to reduce the dimensionality of the pursuit eye movement and express it as a predicted firing rate, in the same units as the actual firing rate.

We applied Equations 2 and 3 to the population averages obtained from our data to generate estimates of the time courses of $R_{N N}$ and $\sigma_{B S}^{2}$, shown in Figure 6 . The data show that the average variance of the eye movement computed using the inverse model prediction of Equation 1 (Fig. $6 \mathrm{~A}$ ), the average variance of actual PC firing (Fig. 6C), and their ratio (Fig. 6E) all have complex time courses that depend on the speed of target (and eye) motion. In general, each variable is larger for higher speeds of target motion. In contrast, the time course and values of the average neuronbehavior correlation are almost identical across target speeds (Fig. 6B). Note that there is remarkably little variance reduction between the firing of individual PCs and the eye movements, the latter as reflected in the surrogate of eye movement provided by the inverse model. Even during the initiation of pursuit, the variance of the eye movement is at least half of the variance of the PC firing rate (Fig. $6 E$ ).

Equation 2 estimates that the average neuron-neuron correlations will show a peak during the initiation of pursuit and then settle to a considerably lower value during steady-state pursuit (Fig. 6D). The estimated neuron-neuron correlations are similar across target speeds during pursuit initiation but are related to target speed during steady-state pursuit. Equation 3 estimates that the variance of the noise added downstream from the cerebellum is relatively small during the initiation of pursuit (Fig. $6 F$ ). At the time of the peak in $R_{N B}, 150 \mathrm{~ms}$ after the onset of target
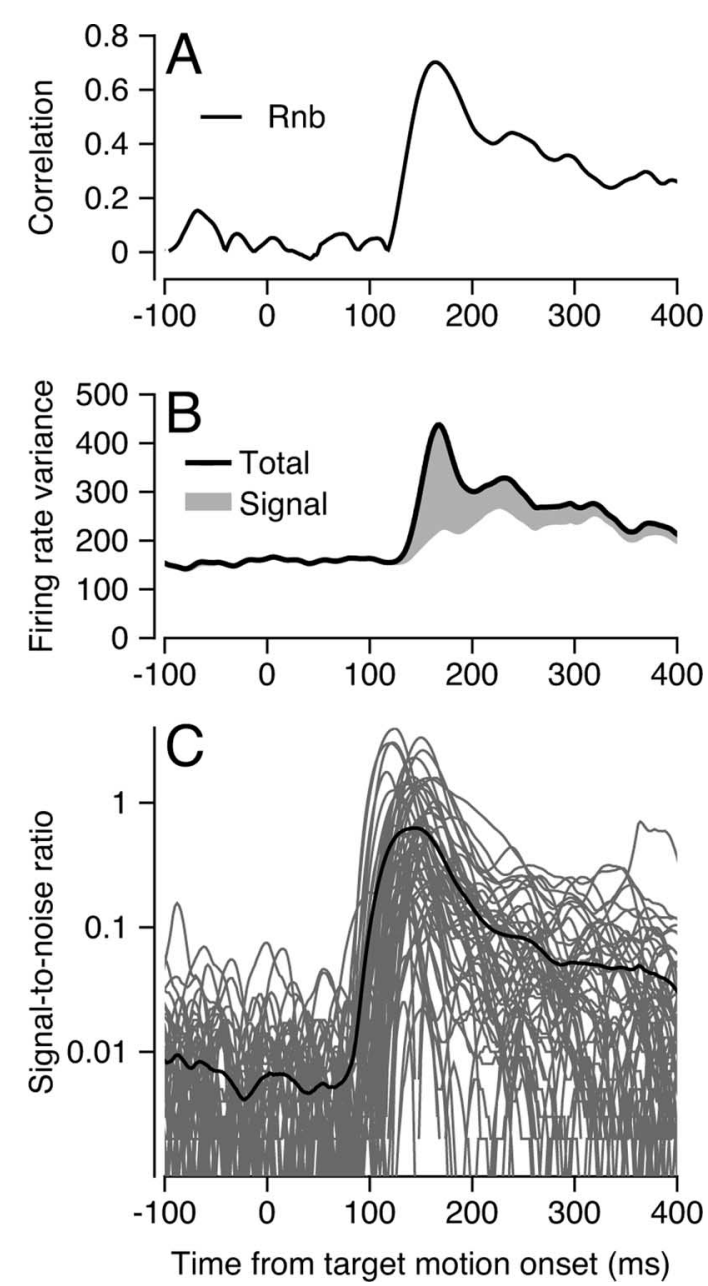

Figure 5. Dynamic modulation of signal and noise in $\mathrm{PC}$ firing. $\boldsymbol{A}$, Time course of neuronbehavior correlation (Rnb) for the example PC from Figures 1 and 2. $\boldsymbol{B}$, The bold trace and the gray area show the time courses of total trial-by-trial variance in firing rate and eye movementrelated variance for the $P C$ in Figures 1 and 2. C, Black and gray traces show the average time course of the signal-to-noise ratio and the individual data for all $51 \mathrm{PCs}$ in our sample. Data are for target motion at $30 \%$ s.

motion (Figs. $6 B, D, F$, vertical dashed lines), the estimated variance of downstream noise is essentially at its baseline of $<8 \%$ of the average variation of PC firing. By the end of the first $100 \mathrm{~ms}$ of pursuit, $200 \mathrm{~ms}$ after the onset of target motion, the estimated downstream variance reaches $\sim 10,30$, and $40 \%$ of the variance of PC firing for target motion at 10,20 , and $30 \%$ s. The estimated downstream noise accumulates further as time proceeds and depends strongly on the speed of eye and target motion during steady-state pursuit.

\section{Neuron-neuron correlations between PCs}

To test the estimates of Figure $6 D$ about neuron-neuron correlations, we recorded from three pairs of PCs in one monkey. The pairs were recorded on separate electrodes at sites that probably were at least $0.5 \mathrm{~mm}$ apart. In Figure $7 A-C$, the red and black traces show the time course of $R_{N B}$ for each of the two neurons in the pair, and the solid blue trace shows the time course of $R_{N N}$. For each pair, the value of $R_{N N}$ reached a peak of $0.25-0.5$ at the same time as the peak of the $R_{N B}$ and declined to values that were not clearly different from zero. The average value of $R_{N N}$ across the three pairs of neurons (Fig. $7 D$, blue line) agreed well with the prediction of Equation 2, computed from the average data across 
the six PCs (Fig. $7 D$, magenta line). The values of $R_{N N}$ were not different from zero at any time during the responses in standard shuffled controls.

Figure $6 D$ allows estimates of the average neuron-neuron correlation, but for pursuit initiation, our data also suggest a way to predict the neuron-neuron correlation between individual pairs of neurons not recorded simultaneously. The measured value of $R_{N N}$ for each of the three pairs of neurons we recorded agreed well during pursuit initiation with the theoretical prediction, confirmed with computer simulation, that in the absence of downstream sources of variance, $R_{N N}$ should be equal to the product of the two values of $R_{N B}$ (Fig. 7A-C, dotted curves). If we consider each possible pair of neurons chosen from the 51 PCs we recorded individually, then Figure $7 E$ shows the predicted neuron-neuron correlation during pursuit initiation in terms of the $R_{N B}$ for the two neurons; the curves are contours that indicate the expected values of $R_{N N}$ for each pair of values of $R_{N B}$. The three pairs we have recorded simultaneously (six large symbols) are distributed across the space of our larger sample, indicating that we sampled a reasonable range of possible pairs.

\section{Discussion}

We found that trial-by-trial variation in the simple spike responses of individual PCs in the floccular complex of the cerebellum is highly predictive of variation in smooth eye movements during the initiation of pursuit. The predictive value declines as pursuit transitions into steadystate performance. We think the high values of neuron-behavior correlation during the initiation of pursuit are remarkable: we knew that the firing of PCs varied substantially from trial to trial (Stone and Lisberger, 1990; Fortier et al., 1993), but we had expected that the variation would be independent across PCs so that the noise emanating from the cerebellum would be reduced dramatically by averaging (Shadlen and Newsome, 1994; Abbott and Dayan, 1999; Averbeck et al., 2006). We also knew that the smooth eye movement evoked during the initiation of pursuit varied from trial to trial, but we were tempted to subscribe to the well known doctrine that motor variation arises from noise injected at very late stages of motor processing (Jones et al., 2002; van Beers et al., 2004), thereby contaminating a reliable motor command. Our data indicate that both of our preconceived notions were wrong. On any given trial, the eye velocity at the initiation of pursuit is faster or slower than
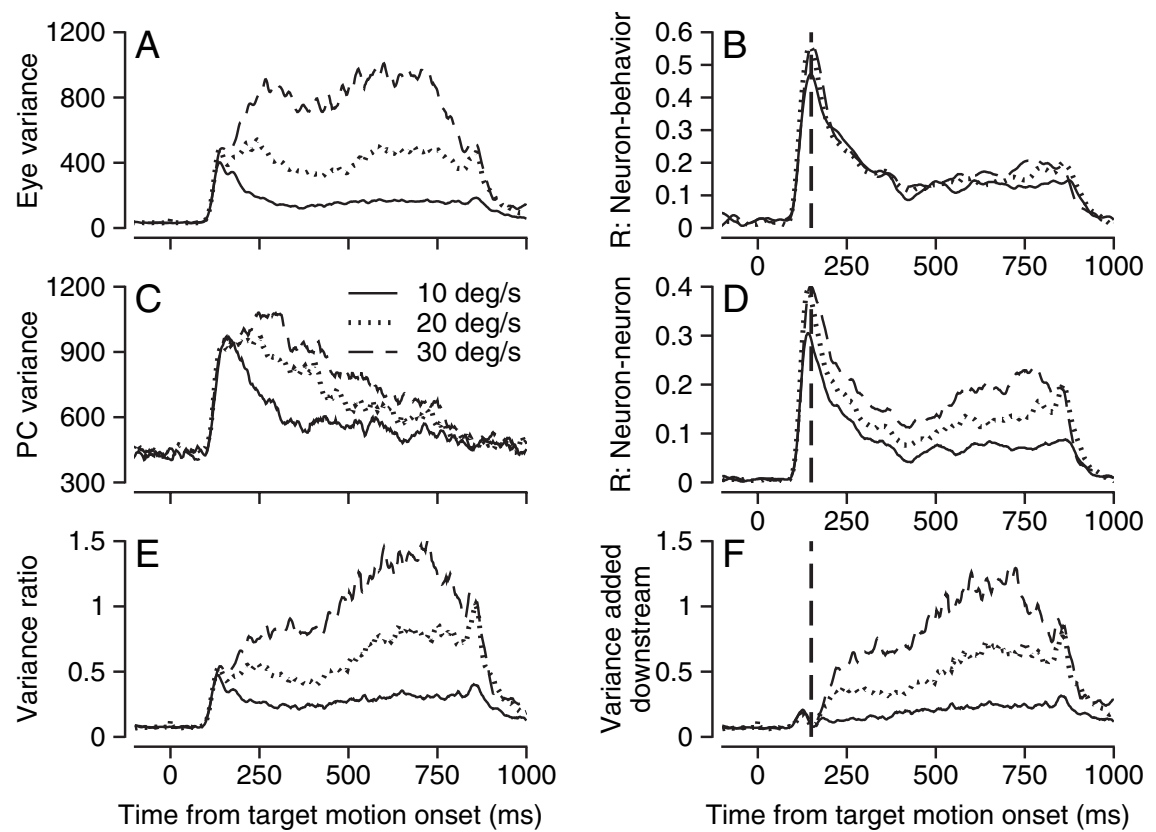

Figure 6. Effect of target/eye speed on the time courses of measured and derived variables. Each trace shows averages across the full sample of PCs. $\boldsymbol{A}$, Measured variance of the eye movement computed using the prediction of the inverse model. $\boldsymbol{B}$, Measured $R_{N B}$. C, Measured variance of actual firing rate. $\boldsymbol{D}$, Predicted $R_{N N}$. $\boldsymbol{E}$, Measured ratio of variance of eye movement to variance of firing rate. $\boldsymbol{F}$, Predicted variance of noise added downstream. Different line styles indicate data obtained for different speeds of target motion, as indicated in the key in $\boldsymbol{C}$.
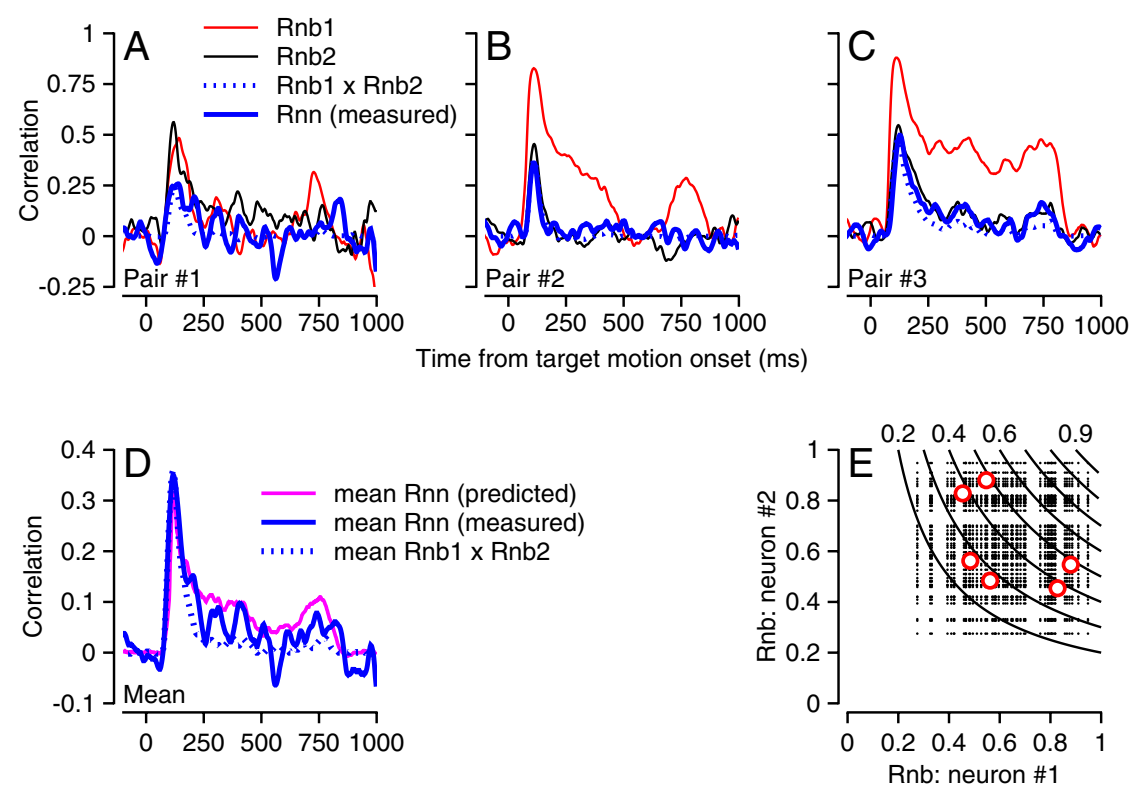

Figure 7. Neuron-neuron correlations. A-C, Time course of neuron-behavior and neuron-neuron correlations for three pairs of PCs. Red and black traces show neuron- behavior correlations for the two neurons in the pair (Rnb1 and Rnb2). Solid blue traces show neuron-neuron correlations (Rnn measured). Dotted blue traces show the product of the two neuron-behavior correlations on a bin-by-bin basis (Rnb1 $\times$ Rnb2). D, Solid blue traces show the average neuron-neuron correlation across all three pairs, the dotted blue trace shows the average product of neuron- behavior correlations across the three pairs, and the magenta trace shows the average value of $R_{N N}$ predicted from Equation 2. $\boldsymbol{E}$, Curves show contour lines of the predicted $R_{N N}$ for pairs of PCs during the initiation of pursuit as a function of the values of $R_{N B}$ in each pair. Small dots show the $51 \times 51$ predictions of $R_{N N}$ during the initiation of pursuit for our sample of 51 neurons recorded separately. The large red circles show $R_{N N}$ for the three pair recordings we made; there are six large red circles to reflect the two possible orders of each pair.

normal because many PCs tend to be slightly more or less active than average. Trial-by-trial variation in behavioral output is already present in the discharge of cerebellar neurons.

To determine the neuron-behavior correlation $\left(R_{N B}\right)$, we re- 
duced the dimensionality of the behavior and transformed it into the same units as the firing rate by applying the standard inverse model of PC firing rate. We determined the best-fitting model for the average response of each PC and applied that model to the eye movements in each trial. As others have found, the model provided superb fits to the average firing rate (Shidara et al., 1993; Leung et al., 2000; Roitman et al., 2005). If it had provided equally good fits to the responses in individual trials, then $R_{N B}$ would have been close to 1 . Thus, the degree to which $R_{N B}$ is $<1$ quantifies the degree of failure of the inverse model to predict the trial-by-trial behavior of PCs. Our analysis has formalized and extended this intuition by using measures of $R_{N B}$ to estimate two factors that would determine how well the average model can predict actual responses in single trials: the magnitude of the correlations in response variation across the population of PCs and the size of any noise that is added downstream from the cerebellum. Our analysis highlights the fallacy of the expectation that the average model would fit perfectly the actual firing rate in each individual trial. If so, then two implausible predictions would follow: no noise is added to sensory-motor processing downstream from PCs and trial-by-trial variations in the firing rate are perfectly correlated across all floccular PCs that contribute to the behavior.

Our measurements predict, based on data, intuition, computer simulation, and theory (see Appendix), that the responses of pairs of PCs are correlated highly during the initiation of pursuit and more weakly during steady-state pursuit. This prediction was supported by recordings from three pairs of PCs, and we found no pairs that contradicted the prediction. The absence of neuron-neuron correlations during fixation for the three pairs of PCs suggests that noise is independent when the eyes are stationary. Because independent noise should be reduced by pooling at the next stage, this may explain why we did not measure noteworthy neuron-behavior correlations during fixation.

We find the agreement of prediction and experiment encouraging, despite the fact that the number of pairs is smaller than we would have liked and too small to make any strong statistical statements. Unfortunately, it proved incredibly difficult to isolate (and hold for the required hour of recording) pairs of PCs that preferred the same axis of pursuit and that were sufficiently responsive to have reasonable values of neuron-behavior correlation. We estimate that $800 \mathrm{~h}$ of experimental time were consumed to obtain the three pairs we have shown. We think that the major difficulty is twofold. First, the geometric organization of the cerebellar cortex mediates against isolating two PCs on one electrode. Second, when two electrodes are introduced into the floccular complex, the nearness of the cerebellar tentorium to the site of recording creates a situation in which moving one electrode invariably moves the cerebellar tissue with respect to the other electrode, reducing the quality of isolation. We expect that new technology will be needed before this experiment will be practical for larger numbers of pairs of PCs.

If firing rate variation is correlated across the population of PCs, then there will be a component of the variation that cannot be eliminated by averaging across the population response (Shadlen and Newsome, 1994; Abbott and Dayan, 1999; Averbeck et al., 2006). The variation in the averaged response becomes a common input to neurons in the next level of sensory-motor processing, creating neuron-neuron correlations that limit the amount of noise reduction as signals pass onward. Thus, the neuron-neuron correlations in PCs (see also De Zeeuw et al., 1997) create a domino effect that moves through the system and becomes a major contributor to variation in the initiation of pursuit. Because some degree of neuron-neuron correlation is present in many brain areas (Gawne and Richmond, 1993; Zohary et al., 1994; Lee et al., 1998), it seems likely that this scenario would be a feature of almost any sensory-motor system. Furthermore, the approach described here could be used at each level of the neural circuit for pursuit or other sensory-motor behaviors to come recursively to a complete understanding of the processing of signal and noise across each individual level of sensorymotor processing.

On the basis of similarities in the noise characteristics for pursuit and perceptual responses guided by visual motion, Osborne et al. (2005) suggested that noise in the visual motion system leads to sensory estimates of target direction and speed that vary from trial to trial, and that the motor system follows these erroneous commands perfectly. In agreement with their suggestion, our analysis predicts that essentially no variation is added in the first $60 \mathrm{~ms}$ to the pursuit commands after they emanate from the cerebellum. Thus, almost all of the variation in this earliest interval of pursuit arises at or before the PCs. Furthermore, the strength of correlated variation in our three pairs of PCs during the initiation of pursuit would be expected if a single signal that varies from trial to trial is distributed widely as a common input to many PCs: we suggest that this signal might arise in the visual motion system. Finally, there is good agreement between the time course of visual motion drive for pursuit and the time course of the predicted neuron-neuron correlations (Lisberger and Westbrook, 1985; Lisberger et al., 1987). Both are largest during the initiation of pursuit, when visual motion signals provide the most prominent drive for pursuit. Later in the response, visual motion inputs become smaller and less important (Lisberger et al., 1987; Morris and Lisberger, 1987), and our analysis predicts that neuron-neuron correlations decline. At the same time, we estimate that the variance of the noise added downstream grows as a function of time during steady-state pursuit and is "signal dependent" in that its amplitude depends on the speed of eye movement. These properties are expected of motor noise (Jones et al., 2002; van Beers et al., 2004). Our data do not predict where the noise is added, but the fact that our analysis is based on the signal and noise in the spike trains of PCs means that the noise must be added downstream from the generation of spikes in PCs. Our data do not exclude the possibility that some of the noise might arise from the failure of the highest frequency simple spike action potentials recorded at the soma of the PC to propagate down its axon to cerebellar target neurons (Khaliq and Raman, 2005; Monsivais et al., 2005).

The dynamic modulation of signal and noise in individual PCs provides some new insights into cerebellar function. Our findings raise the possibility that there is a transition from highly covariant cerebellar responses during movement initiation to more independent responses later on, and this prediction was confirmed in our small sample of pairs of PCs. The exact degree of correlation across the population of PCs may be important for the operation of the circuit: excessive variation in $\mathrm{PC}$ responses is associated with motor deficits (Hoebeek et al., 2005; Walter et al., 2006), whereas correlated variation in firing across the population may enhance the effect of PCs on their target neurons by engaging a postinhibitory rebound (Aizenman and Linden, 1999; Sekirnjak et al., 2003). At the same time, persistent strong correlations in firing across the population of PCs could create synchronous oscillations that would present a hazard to stability in motor networks and possibly result in ataxia (Cheron et al., 2005). The balance between correlation and asynchrony may be adaptive: the cerebellar micro-circuit might benefit from the ad- 
vantages of highly correlated responses early in pursuit and purposively degrade the correlation later in the response. This kind of dynamic modulation of variation, covariation, and noise may be imperative for generation of precise outputs by a noisy brain.

\section{Appendix}

In Results, we make predictions about the degree of noise reduction that is obtained by averaging across PCs and the amount of noise that is added downstream from the PCs. The equations for making these predictions were obtained from analytical solution and computer simulations of a model in which the firing rate of the ith PC at a particular time is defined as follows:

$$
f r_{i}=\sqrt{R_{N N}} \nu_{i}+\sqrt{\left(1-R_{N N}\right)} \rho_{i}
$$

Here, the $\rho$ and $\nu$ are random variables with mean equal to zero and variance equal to $\sigma_{F R}^{2}$; relaxing these assumptions to allow the firing rates of different PCs to have different means and variances precludes a full analytical solution, but simulation showed that the conclusions of our analysis did not change under more heterogeneous conditions. $R_{N N}$ is a free parameter that determines the degree to which firing rates at a particular time are correlated across PCs $\left(R_{N N}=1\right.$ implies PCs are perfectly correlated; $R_{N N}=$ 0 implies the firing of different PCs is independent of each other). This form of the model lacks any explicit correlations across time and therefore can be solved for the static case and then applied to data for each particular time. For any given time and trial, $\nu$ is drawn once, multiplied by $\sqrt{R_{N N}}$, and assigned to all PCs, whereas $\rho$ is drawn separately for each PC and multiplied by $\sqrt{\left(1-R_{N N}\right)}$. This creates two components of PC firing. The first component is perfectly correlated across neurons on each individual trial but uncorrelated across trials and has a variance of $R_{N N} \sigma_{F R}^{2}$. The second component is uncorrelated across both neurons and trials and has a variance of $\left(1-R_{N N}\right) \sigma_{F R}^{2}$.

We assume that we can write the decoded output of the pursuit system as follows:

$$
E Y E=\frac{\sum_{i=1}^{M} f r_{i}}{M}+\beta
$$

where $M$ is the number of PCs and $\beta$ is a random variable that is added downstream in the brainstem after the firing of all PCs has been averaged. The downstream signal adds noise with a variance of $\sigma_{B S}^{2}$. In Equation 5, EYE has the same units as firing rate and is equivalent to the predicted firing rate derived for each PC using the inverse model from Equation 1. Our analysis depends on the single assumption of Equation 5 that the population response of floccular PCs is converted into a command for smooth eye movement by simply averaging the population response. Other methods, such as maximum likelihood, vector average, winner-takeall and Bayesian, least-squares, and optimal linear estimators, have been postulated for decoding the response of neural populations (Salinas and Abbott, 1994), and it is clear that noise reduction and the effects of correlated activity will depend on the specifics of the decoding algorithm (Abbott and Dayan, 1999; Salinas and Sejnowski, 2000, 2001; Shamir and Sompolinsky, 2006). However, simple decoding methods such as averaging the population response are likely to be implemented in the brain in cases when all of the neurons are monotonically tuned for the parameter of interest, as PCs are for eye velocity (Lisberger and Fuchs, 1978; Robinson and Fuchs, 2001). Indeed, previous analyses of PCs have all supported the idea that their responses simply are averaged to create a command for eye velocity (Krauzlis and Lisberger, 1994; Coltz et al., 2000; Krauzlis, 2000), in agreement with the key assumption made by Equation 5 .

Variances sum. So, we can write the trial-by-trial variance of the eye movement as follows:

$$
\sigma_{E Y E}^{2}=R_{N N} \sigma_{F R}^{2}+\frac{\left(1-R_{N N}\right) \sigma_{F R}^{2}}{M}+\sigma_{B S}^{2}
$$

$M$ is present in only the second term of Equation 6 because the variance of the correlated component of firing rate will not be reduced by averaging across neurons within an individual trial, whereas the variance of the uncorrelated component of firing rate will be reduced in proportion to the number of neurons, $M$. If we divide both sides of Equation 6 by the variance of PC firing, we get the following:

$$
\frac{\sigma_{E Y E}^{2}}{\sigma_{F R}^{2}}=R_{\mathrm{NN}}+\frac{\left(1-R_{\mathrm{NN}}\right)}{M}+\frac{\sigma_{B S}^{2}}{\sigma_{F R}^{2}}
$$

Equation 7 is our first analytical result and will be used later.

We next derive an analytical expression for $R_{N B}$, the neuronbehavior correlations measured here. The correlation between two random variables, $X$ and $Y$, is defined as follows:

$$
R=\frac{E(X \cdot Y)}{\sqrt{E(X \cdot X) E(Y \cdot Y)}}
$$

We can convert this to our notation and use it to represent and predict the neuron-behavior correlations we have computed from our data:

$$
R_{N B}=R\left(f r_{i}, E Y E\right)=\frac{E\left(f r_{i} \cdot E Y E\right)}{\sqrt{E\left(f r_{i} \cdot f r_{i}\right) E(E Y E \cdot E Y E)}}
$$

Next, we compute each of the expected values $E(X, Y)$ in Equation 9:

$$
\begin{aligned}
E\left(f r_{i} \cdot E Y E\right) & =E\left[\left(\sqrt{R_{N N}} \nu_{i}+\sqrt{\left(1-\mathrm{R}_{\mathrm{NN}}\right)} \rho_{\mathrm{i}}\right)\right. \\
& \left.\left(\frac{1}{\mathrm{M}}\left[\sum\left(\sqrt{R_{\mathrm{NN}}} \nu_{\mathrm{j}}+\sqrt{\left(1-\mathrm{R}_{\mathrm{NN}}\right)} \rho_{\mathrm{j}}\right)\right]+\beta\right)\right]
\end{aligned}
$$

The fact that all the $\nu_{i}=\nu_{j}$ on a given trial allows us to bring almost all the terms outside the summation in Equation 10a, yielding the following:

$$
\begin{array}{r}
E\left(f r_{i} \cdot E Y E\right)=E\left[\left(\sqrt{R_{N N}} \nu_{i}+\sqrt{\left(1-R_{N N}\right)} \rho_{i}\right)\right. \\
\left.\left(\frac{1}{M}\left[M \sqrt{R_{N N}} \nu_{i}+\sqrt{1-R_{N N}} \sum_{j} \rho_{j}\right]+\beta\right)\right]
\end{array}
$$

and

$$
\begin{aligned}
E\left(f r_{i} \cdot E Y E\right)=E[ & {\left[\sqrt{R_{N N}} \nu_{i}+\sqrt{\left(1-R_{N N}\right)} \rho_{i}\right) } \\
& \left.\left(\sqrt{R_{N N}} \nu_{i}+\frac{\sqrt{1-R_{N N}}}{M} \sum_{j} \rho_{j}+\beta\right)\right]
\end{aligned}
$$


If we evaluate Equation 10c by multiplying the terms within the large parentheses, almost all of the terms have expected values of zero because they multiply two random variables that have independent distributions with means equal to zero. This leaves two terms that contain the same random variable twice, leading to the following:

$$
E\left(f r_{i} \cdot E Y E\right)=E\left[R_{N N} \nu_{i}^{2}+\frac{1}{M}\left(1-R_{N N}\right) \rho_{i}^{2}\right]
$$

We use the identities: $E(X+Y)=E(X)+E(Y) ; E(c X)=c E(X)$; and $E\left(X^{2}\right)=\sigma_{X}^{2}$. Because $E\left(\nu_{i}^{2}\right)=E\left(\rho_{i}^{2}\right)=\sigma_{f r}^{2}$, we can convert Equation 11 to the following:

$$
E\left(f r_{i} \cdot E Y E\right)=\sigma_{F R}^{2}\left(R_{N N}+\frac{1}{M}\left(1-R_{N N}\right)\right)
$$

Similarly, the expected value of a zero-mean random variable crossed on itself is the variance of the random variable, leading to the following:

$$
E\left(f r_{i} \cdot f r_{i}\right)=\sigma_{F R}^{2}\left(R_{N N}+\left(1-R_{N N}\right)\right)=\sigma_{F R}^{2}
$$

and

$$
E(E Y E \cdot E Y E)=\sigma_{F R}^{2}\left(R_{N N}+\frac{\left(1-R_{N N}\right)}{M}\right)+\sigma_{B S}^{2}
$$

Now, we can substitute Equations 12-14 into Equation 9 to obtain an expression for neuron-behavior correlations:

$$
R_{N B}=\frac{\frac{1}{M}\left(1-R_{N N}\right)+R_{N N}}{\sqrt{\frac{1}{M}\left(1-R_{N N}\right)+R_{N N}+\frac{\sigma_{B S}^{2}}{\sigma_{F R}^{2}}}}
$$

Note that $R_{N B}$ is equal to $\sqrt{1 / M}$ even if $R_{N N}$ and $\sigma_{B S}^{2}$ are zero. Thus, a large value of $R_{N B}$ predicts large values of $R_{N N}$ only if a substantial number of PCs contribute to the behavior, as seems likely in the brain.

Equations 7 and 15 describe two variables we can measure in our data: the neuron-behavior correlations $\left(R_{N B}\right)$ and the ratio of the trial-by-trial variances of the eye movement and the firing rate of PCs $\left(\sigma_{E Y E}^{2} / \sigma_{f r}^{2}\right)$. The equations have only three unknowns: the number of PCs $(M)$, the neuron-neuron correlation among PCs $\left(R_{N N}\right)$, and the variance of the noise added downstream from averaging the responses of PCs $\left(\sigma_{B S}^{2}\right)$. If we assume that the value of $M$ is fairly large, then Equations 7 and 15 operate as two solvable equations in two unknowns, allowing us to predict the time course of $R_{N N}$ and $\sigma_{B S}^{2}$ from the measurements we have made. Equations 2 and 3 are solutions under the assumption that the number of PCs $(M)$ is greater than $\sim 40$ so that any term with $M$ in the denominator is effectively zero.

Figure 8 summarizes the results of simulating the model described by Equations 7 and 15. In Figure 8, $A$ and $B$, the simulation was run with 100 model neurons. The value of $\sigma_{F R}^{2}$ was one, and both $R_{N N}$ and $\sigma_{B S}^{2}$ were varied in steps of 0.1 between 0.1 and 0.9 . The points along each different curve show the results of the simulation for different values of $R_{N N}$, and each set of points is plotted as a function of $\sigma_{B S}^{2}$. Neuron-behavior correlation decreased as a function of $\sigma_{B S}^{2}$ and increased as a function of $R_{N N}$ (Fig. $8 A$ ). The ratio of the variances of the eye movement to PC firing rate increased as a function of $\sigma_{B S}^{2}$ and $R_{N N}$ (Fig. $8 B$ ). The continuous traces show the results of the analytical solution,
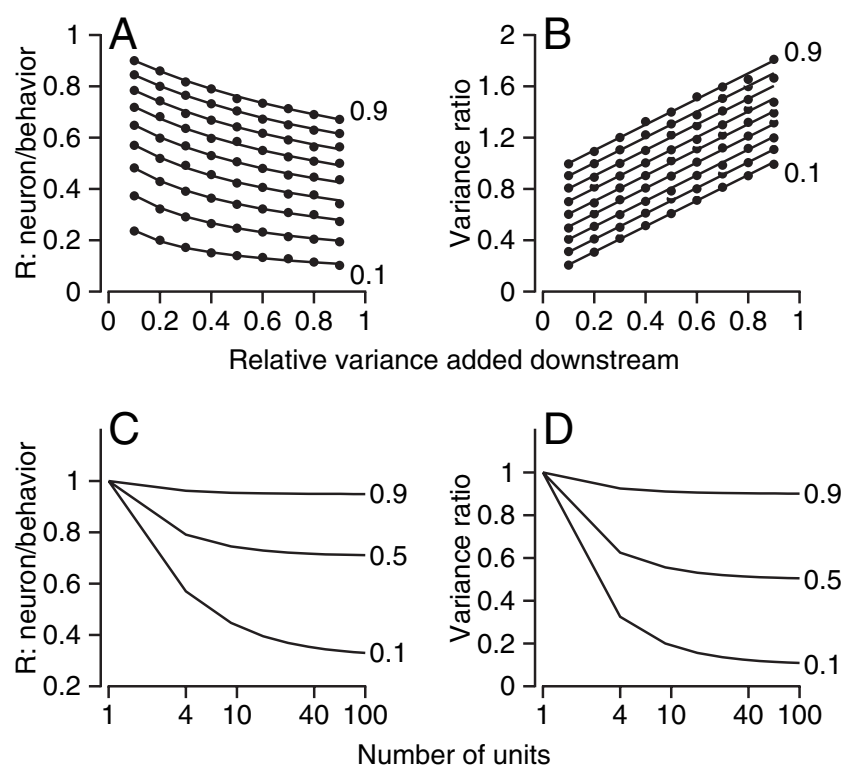

Figure 8. Computer simulations showing the effect of neuron-neuron correlations and downstream noise on neuron-behavior correlations and the amount of noise reduction achieved between the firing of $\mathrm{PCs}$ and the evoked eye movement. $\boldsymbol{A}, \boldsymbol{B}, R_{N B}(\boldsymbol{A})$ and the ratio of eye movement variance to firing rate variance $(\boldsymbol{B})$ are plotted as a function of the variance of noise added downstream. Eye movement variance was computed as the variance across trials of the firing rate predicted by the inverse model. From bottom to top, the curves and associated symbols plot results for analytical solution (curves) or simulation (symbols) as the value of $R_{N N}$ is increased from 0.1 to 0.9 in steps of 0.1 . C, D, The same two variables are plotted as a function of the number of model $P C s$, for the analytical solution given in the Appendix. The variance of noise added downstream was zero, and the three curves in each graph show solutions when $R_{N N}$ was $0.1,0.5$, or 0.9 .

agreeing perfectly with the results of the simulations. Figure 8, $C$ and $D$, shows the effect of varying the number of PCs in the simulation on the neuron-behavior correlations and the variance ratio, for three values of $R_{N N}$ when $\sigma_{B S}^{2}$ was 0 . The curves come close to asymptotic behavior if the model contains $\sim 40$ PCs, as expected from previous analyses (Shadlen et al., 1996). For the simulations in Figure 8, all model PCs had the same values of $\sigma_{F R}^{2}$ and response magnitude, and the responses were randomized across model neurons and trials. The same findings applied when we extended the simulations to the more natural case in which different model PCs have different values of $\sigma_{F R}^{2}$, different response magnitudes, or even different values of $R_{N N}$.

\section{References}

Abbott LF, Dayan P (1999) The effect of correlated variability on the accuracy of a population code. Neural Comput 11:91-101.

Aizenman CD, Linden DJ (1999) Regulation of the rebound depolarization and spontaneous firing patterns of deep nuclear neurons in slices of rat cerebellum. J Neurophysiol 82:1697-1709.

Averbeck BB, Latham PE, Pouget A (2006) Neural correlations, population coding and computation. Nat Rev Neurosci 7:358-366.

Calvin WH, Stevens CF (1967) Synaptic noise as a source of variability in the interval between action potentials. Science 155:842-844.

Calvin WH, Stevens CF (1968) Synaptic noise and other sources of randomness in motoneuron interspike intervals. J Neurophysiol 31:574-587.

Carmena JM, Lebedev MA, Henriquez CS, Nicolelis MA (2005) Stable ensemble performance with single-neuron variability during reaching movements in primates. J Neurosci 25:10712-10716.

Cheron G, Servais L, Wagstaff J, Dan B (2005) Fast cerebellar oscillation associated with ataxia in a mouse model of Angelman syndrome. Neuroscience 130:631-637. 
Coltz JD, Johnson MT, Ebner TJ (2000) Population code for tracking velocity based on cerebellar Purkinje cell simple spike firing in monkeys. Neurosci Lett 296:1-4.

Davids, K, Bennett S, Newell KM (2006) Movement system variability, xii. Champaign, IL: Human Kinetics.

De Zeeuw CI, Koekkoek SK, Wylie DR, Simpson JI (1997) Association between dendritic lamellar bodies and complex spike synchrony in the olivocerebellar system. J Neurophysiol 77:1747-1758.

Fortier PA, Smith AM, Kalaska JF (1993) Comparison of cerebellar and motor cortex activity during reaching: directional tuning and response variability. J Neurophysiol 69:1136-1149.

Fuchs AF, Robinson DA (1966) A method for measuring horizontal and vertical eye movement chronically in the monkey. J Appl Physiol 21:1068-1070.

Gawne TJ, Richmond BJ (1993) How independent are the messages carried by adjacent inferior temporal cortical neurons? J Neurosci 13:2758 -2771.

Hoebeek FE, Stahl JS, van Alphen AM, Schonewille M, Luo C, Rutteman M, van den Maagdenberg AM, Molenaar PC, Goossens HH, Frens MA, De Zeeuw CI (2005) Increased noise level of Purkinje cell activities minimizes impact of their modulation during sensorimotor control. Neuron 45:953-965.

Jones KE, Hamilton AF, Wolpert DM (2002) Sources of signaldependent noise during isometric force production. J Neurophysiol 88:1533-1544.

Khaliq ZM, Raman IM (2005) Axonal propagation of simple and complex spikes in cerebellar Purkinje neurons. J Neurosci 25:454-463.

Krauzlis RJ (2000) Population coding of movement dynamics by cerebellar Purkinje cells. NeuroReport 11:1045-1050.

Krauzlis RJ, Lisberger SG (1994) Simple spike responses of gaze velocity Purkinje cells in the floccular lobe of the monkey during the onset and offset of pursuit eye movements. J Neurophysiol 72:2045-2050.

Lee D, Port NL, Kruse W, Georgopoulos AP (1998) Variability and correlated noise in the discharge of neurons in motor and parietal areas of the primate cortex. J Neurosci 18:1161-1170.

Leung HC, Suh M, Kettner RE (2000) Cerebellar flocculus and paraflocculus Purkinje cell activity during circular pursuit in monkey. J Neurophysiol 83:13-30.

Lisberger SG, Fuchs AF (1978) Role of primate flocculus during rapid behavioral modification of vestibuloocular reflex. I. Purkinje cell activity during visually guided horizontal smooth-pursuit eye movements and passive head rotation. J Neurophysiol 41:733-763.

Lisberger SG, Pavelko TA (1986) Vestibular signals carried by pathways subserving plasticity of the vestibulo-ocular reflex in monkeys. J Neurosci 6:346-354

Lisberger SG, Westbrook LE (1985) Properties of visual inputs that initiate horizontal smooth pursuit eye movements in monkeys. J Neurosci 5:1662-1673.

Lisberger SG, Morris EJ, Tychsen L (1987) Visual motion processing and sensory-motor integration for smooth pursuit eye movements. Annu Rev Neurosci 10:97-129.

Medina JF, Carey MR, Lisberger SG (2005) The representation of time for motor learning. Neuron 45:157-167.

Monsivais P, Clark BA, Roth A, Hausser M (2005) Determinants of action potential propagation in cerebellar Purkinje cell axons. J Neurosci 25:464-472.

Morris EJ, Lisberger SG (1987) Different responses to small visual errors during initiation and maintenance of smooth-pursuit eye movements in monkeys. J Neurophysiol 58:1351-1369.

Osborne LC, Lisberger SG, Bialek W (2005) A sensory source for motor variation. Nature 437:412-416.

Pouget A, Dayan P, Zemel R (2000) Information processing with population codes. Nat Rev Neurosci 1:125-132.
Ramachandran R, Lisberger SG (2005) Normal performance and expression of learning in the vestibulo-ocular reflex (VOR) at high frequencies. J Neurophysiol 93:2028-2038.

Rashbass C (1961) The relationship between saccadic and smooth tracking eye movements. J Physiol (Lond) 159:326-338.

Robinson FR, Fuchs AF (2001) The role of the cerebellum in voluntary eye movements. Annu Rev Neurosci 24:981-1004.

Roitman AV, Pasalar S, Johnson MT, Ebner TJ (2005) Position, direction of movement, and speed tuning of cerebellar Purkinje cells during circular manual tracking in monkey. J Neurosci 25:9244-9257.

Salinas E, Abbott LF (1994) Vector reconstruction from firing rates. J Comput Neurosci 1:89-107.

Salinas E, Sejnowski TJ (2000) Impact of correlated synaptic input on output firing rate and variability in simple neuronal models. J Neurosci 20:6193-6209.

Salinas E, Sejnowski TJ (2001) Correlated neuronal activity and the flow of neural information. Nat Rev Neurosci 2:539-550.

Sekirnjak C, Vissel B, Bollinger J, Faulstich M, du Lac S (2003) Purkinje cell synapses target physiologically unique brainstem neurons. J Neurosci 23:6392-6398.

Shadlen MN, Newsome WT (1994) Noise, neural codes and cortical organization. Curr Opin Neurobiol 4:569-579.

Shadlen, MN, Britten KH, Newsome WT, Movshon JA (1996) A computational analysis of the relationship between neuronal and behavioral responses to visual motion. J Neurosci 16:1486-1510.

Shamir M, Sompolinsky H (2006) Implications of neuronal diversity on population coding. Neural Comput 18:1951-1986.

Shidara M, Kawano K, Gomi H, Kawato M (1993) Inverse-dynamics model eye movement control by Purkinje cells in the cerebellum. Nature 365:50-52.

Softky WR, Koch C (1993) The highly irregular firing of cortical cells is inconsistent with temporal integration of random EPSPs. J Neurosci 13:334-350.

Stein RB, Gossen ER, Jones KE (2005) Neuronal variability: noise or part of the signal? Nat Rev Neurosci 6:389-397.

Stone LS, Lisberger SG (1990) Visual responses of Purkinje cells in the cerebellar flocculus during smooth-pursuit eye movements in monkeys. I. Simple spikes. J Neurophysiol 63:1241-1261.

Takemura A, Inoue Y, Gomi H, Kawato M, Kawano K (2001) Change in neuronal firing patterns in the process of motor command generation for the ocular following response. J Neurophysiol 86:1750-1763.

Thier P, Ilg UJ (2005) The neural basis of smooth-pursuit eye movements. Curr Opin Neurobiol 15:645-652.

Todorov E, Jordan MI (2002) Optimal feedback control as a theory of motor coordination. Nat Neurosci 5:1226-1235.

van Beers RJ, Baraduc P, Wolpert DM (2002) Role of uncertainty in sensorimotor control. Philos Trans R Soc Lond B Biol Sci 357:1137-1145.

van Beers RJ, Haggard P, Wolpert DM (2004) The role of execution noise in movement variability. J Neurophysiol 91:1050-1063.

von Neumann J (1956) Probabilistic logics and the synthesis of reliable organisms from unreliable components. In: Automata studies (Shannon CE, McCarthy J, Ashby WR, eds), pp 43-98. Princeton, NJ: Princeton UP.

Walter JT, Alvina K, Womack MD, Chevez C, Khodakhah K (2006) Decreases in the precision of Purkinje cell pacemaking cause cerebellar dysfunction and ataxia. Nat Neurosci 9:389-397.

White JA, Rubinstein JT, Kay AR (2000) Channel noise in neurons. Trends Neurosci 23:131-137.

Zohary E, Shadlen MN, Newsome WT (1994) Correlated neuronal discharge rate and its implications for psychophysical performance. Nature 370:140-143. 